\title{
On the Distribution Functions of the Range and Quasi-range for the Extended Type I Generalized Logistic Distribution
}

\author{
A. K. Olapade \\ Obafemi Awolowo University
}

\begin{abstract}
In this paper, we obtain the distribution functions of the range and the quasi-range of the random variables arising from the extended type I generalized logistic distribution.
\end{abstract}

Keywords. Extended type I generalized logistic distribution; order statistics; Quasi-range and range.

\section{Introduction}

The probability density function (pdf) of the standard logistic distribution is

$$
f_{X}(x)=\frac{e^{-x}}{\left(1+e^{-x}\right)^{2}}, \quad-\infty<x<\infty,
$$

and hence its cumulative distribution function (cdf) is

$$
F_{X}(x)=\left(1+e^{-x}\right)^{-1}, \quad-\infty<x<\infty .
$$

The shape of this distribution is similar to that of normal distribution has made it to be preferred to normal distribution by some researchers like Berkson (1944, 1950, 1953), Berkson and Hodges (1960), etc. Ojo (1989) used the logistic model to analyze some social data sets. Researchers have been working on order statistics from the logistic distribution for a long time. It was considered in Plackett (1958), Birnbaum and Dudman (1963), Tarter and Clark (1965), Shah (1966, 1970). Gupta et al. (1967) obtained best 
unbiased estimators for the parameters of the logistic distribution using order statistics. It is well known that the range and quasi-range are important statistics which are defined based on order statistics. Gupta and Shah (1965) obtained the distribution of the range from the logistic distribution while Malik (1980) obtained the distribution function of the quasi-range from the logistic distribution. In recent times, researchers have focused more on generalizing pdfs with the aim of making the functions to be more robust and applicable to model different types of data. The logistic distribution has enjoyed the practice of generalization in many forms as could be seen in George and Ojo (1980), Balakrishnan and Leung (1988a), Wu et al. (2000), Olapade $(2004,2005,2006)$. Though, many works have been done on the order statistics from the logistics distribution, many of its various generalizations of the distribution have not enjoyed such privilege. Balakrishnan and Leung (1988a) studied order statistics from the type I generalized logistic distribution while Balakrishnan and Leung (1988b) obtained the means, variances and covariances of the order statistics, BLUE's for the type I generalized logistic distribution. In this paper, we shall obtain the distributions of the range and quasi-range of the extended type I generalized logistic distribution with pdf

$$
f_{X}(x ; \lambda, p)=\frac{p \lambda^{p} e^{-x}}{\left(\lambda+e^{-x}\right)^{p+1}}, \quad-\infty<x<\infty, p>0, \lambda>0
$$

and cdf

$$
F_{X}(x ; \lambda, p)=\frac{\lambda^{p}}{\left(\lambda+e^{-x}\right)^{p}}, \quad-\infty<x<\infty, p>0, \lambda>0 .
$$

Some properties and application of this distribution were presented in Olapade (2009) who obtained the distribution of the $r$ th order statistics and established the pdfs of the maximum and minimum order statistics in a random sample.

\section{Distribution of the Range}

Given a set of random variables $X_{1}, X_{2}, \ldots, X_{n}$ of size $n$ coming from the extended type I generalized logistic distribution, let $X_{1: n} \leqslant X_{2: n} \leqslant \cdots \leqslant$ $X_{n: n}$ be the corresponding order statistics. Let $F_{X_{r: n}}(x)$ and $f_{X_{r: n}}(x), r=$ $1,2, \ldots, n$ be the cdf and pdf of the $r$ th order statistics $X_{r: n}$ respectively. 
David (1970) obtained the pdf of $X_{r: n}$ as

$$
f_{X_{r: n}}(x)=\frac{1}{B(r, n-r+1)}[F(x)]^{r-1}[1-F(x)]^{n-r} f(x) .
$$

Let us define the sample range $W_{n}$ by $W_{n}=X_{n: n}-X_{1: n}$. The cdf of $W_{n}$ can be written as (Gupta and Shah, 1965)

$$
\operatorname{Pr}\left(W_{n} \leqslant w\right)=n \int_{-\infty}^{+\infty}\{F(x+w)-F(x)\}^{n-1} f(x) d x .
$$

By expanding $\{F(x+w)-F(x)\}^{n-1}$, we have

$$
\operatorname{Pr}\left(W_{n} \leqslant w\right)=n \sum_{k=0}^{n-1}\left(\begin{array}{c}
n-1 \\
k
\end{array}\right) \int_{-\infty}^{+\infty}\{F(x+w)\}^{n-1-k}\{-F(x)\}^{k} f(x) d x .
$$

Substituting (1) and (2) in (3) we have

$$
\begin{aligned}
\operatorname{Pr}\left(W_{n} \leqslant w\right)= & n \sum_{k=0}^{n-1}(-1)^{k}\left(\begin{array}{c}
n-1 \\
k
\end{array}\right) \int_{-\infty}^{+\infty}\left\{\frac{\lambda}{\lambda+e^{-w-x}}\right\}^{p(n-1-k)} \\
& \times\left\{\frac{\lambda}{\lambda+e^{-x}}\right\}^{p k} \frac{p \lambda^{p} e^{-x}}{\left(\lambda+e^{-x}\right)^{p+1}} d x \\
= & n p \lambda^{n p} \sum_{k=0}^{n-1}(-1)^{k}\left(\begin{array}{c}
n-1 \\
k
\end{array}\right) \\
& \int_{-\infty}^{+\infty} \frac{e^{-x}}{\left(\lambda+e^{-w-x}\right)^{p(n-1-k)}\left(\lambda+e^{-x}\right)^{p k+p+1}} d x .
\end{aligned}
$$

Let $t=\left(\lambda+a e^{-x}\right)^{-1}$, where $a=e^{-w}$, then

$$
\begin{aligned}
\operatorname{Pr}\left(W_{n} \leqslant w\right)= & n p \lambda^{n p} \sum_{k=0}^{n-1}(-1)^{k}\left(\begin{array}{c}
n-1 \\
k
\end{array}\right) a^{p k+p} \\
& \int_{0}^{\frac{1}{\lambda}} \frac{t^{n p-1}}{\{1+\lambda(a-1) t\}^{p k+p+1}} d t \\
= & n p \lambda^{n p} \sum_{k=0}^{n-1}(-1)^{k}\left(\begin{array}{c}
n-1 \\
k
\end{array}\right) a^{p(k+1)} \int_{0}^{\frac{1}{\lambda}} \frac{t^{n p-1}}{(1+b t)^{p k+p+1}} d t \\
= & n p \lambda^{n p} \sum_{k=0}^{n-1}(-1)^{k}\left(\begin{array}{c}
n-1 \\
k
\end{array}\right) a^{p(k+1)} A(k, p, n, \lambda),
\end{aligned}
$$


where $b=\lambda(a-1), v=1+b t$ and

$$
\begin{aligned}
A(k, p, n, \lambda)= & \int_{0}^{\frac{1}{\lambda}} \frac{t^{n p-1}}{(1+b t)^{p k+p+1}} d t \\
= & \frac{-1}{(-b)^{n p}} \int_{1}^{\frac{1+b}{\lambda}} v^{-p k-p-1}(1-v)^{n p-1} d v \\
= & \frac{-1}{(-b)^{n p}} \sum_{j=0}^{n p-1}(-1)^{j}\left(\begin{array}{c}
n p-1 \\
j
\end{array}\right) \int_{1}^{\frac{1+b}{\lambda}} v^{j-p k-p-1} d v \\
= & \frac{-1}{(-b)^{n p}}\left\{(-1)^{p k+p}\left(\begin{array}{c}
n p-1 \\
p k+p
\end{array}\right) \ln \left(1+\frac{b}{\lambda}\right)\right. \\
& \left.+\sum_{j=0, j \neq p k+p}^{n p-1}(-1)^{j}\left(\begin{array}{c}
n p-1 \\
j
\end{array}\right) \frac{\left(\frac{1+b}{\lambda}\right)^{j-p k-p}-1}{j-p k-p}\right\}
\end{aligned}
$$

Substitute for $A(k, p, n, \lambda)$ in (4), we have

$$
\begin{aligned}
\operatorname{Pr}\left(W_{n} \leqslant w\right)= & n p \sum_{k=0}^{n-1}(-1)^{k+1}\left(\begin{array}{c}
n-1 \\
k
\end{array}\right) \\
& a^{p(k+1)} \frac{1}{(1-a)^{n p}}\left\{(-1)^{p k+p}\left(\begin{array}{c}
n p-1 \\
p k+p
\end{array}\right) \ln a\right. \\
& \left.+\sum_{j=0, j \neq p k+p}^{n p-1}(-1)^{j}\left(\begin{array}{c}
n p-1 \\
j
\end{array}\right) \frac{a^{j-p k-p}-1}{j-p k-p}\right\} \\
= & \frac{n p}{\left(1-e^{-w}\right)^{n p}} \sum_{k=0}^{n-1}(-1)^{k+1}\left(\begin{array}{c}
n-1 \\
k
\end{array}\right) \\
& \left\{(-1)^{p k+p-1}\left(\begin{array}{c}
n p-1 \\
p k+p
\end{array}\right) w e^{-w p(k+1)}\right. \\
& \left.+\sum_{j=0, j \neq p k+p}^{n p-1}(-1)^{j}\left(\begin{array}{c}
n p-1 \\
j
\end{array}\right) \frac{e^{-w j}-e^{-w p(k+1)}}{j-p k-p}\right\} .
\end{aligned}
$$


By differentiating the distribution function of the sample range in equation (2.14) with respect to $w$, we derive the pdf of $W_{n}$ as

$$
\begin{aligned}
p(w)= & \frac{n^{2} p^{2} e^{-w}}{\left(1-e^{-w}\right)^{n p+1}} \sum_{k=0}^{n-1}(-1)^{k+1}\left(\begin{array}{c}
n-1 \\
k
\end{array}\right) \\
& \left\{(-1)^{p k+p-1}\left(\begin{array}{c}
n p-1 \\
p k+p
\end{array}\right) w e^{-w p(k+1)}\right. \\
& \left.+\sum_{j=0, j \neq p k+p}^{n p-1}(-1)^{j}\left(\begin{array}{c}
n p-1 \\
j
\end{array}\right) \frac{e^{-w j}-e^{-w p(k+1)}}{j-p k-p}\right\} \\
& +\frac{n p}{\left(1-e^{-w}\right)^{n p}} \sum_{k=0}^{n-1}(-1)^{k+1}\left(\begin{array}{c}
n-1 \\
k
\end{array}\right) \\
& {\left[(-1)^{p k+p-1}\left(\begin{array}{c}
n p-1 \\
p k+p
\end{array}\right)\{1-w p(k+1)\} e^{-w p(k+1)}\right.} \\
& \left.+\sum_{j=0, j \neq p k+p}^{n p-1}(-1)^{j}\left(\begin{array}{c}
n p-1 \\
j
\end{array}\right) \frac{p(k+1) e^{-w p(k+1)}-j e^{-w j}}{j-p k-p}\right] .
\end{aligned}
$$

It could be noted that the $F_{W}(w)$ and $f_{W}(w)$ of the extended type I generalized logistic distribution are free of $\lambda$.

\section{Distribution of the Quasi-range}

The sample $r$ th quasi-range denoted by $W$, is defined as

$$
W=X_{n-r: n}-X_{r+1: n}, \quad r=0,1, \ldots, \frac{n-1}{2},
$$

where $n$ is odd. Thus the joint pdf of $X_{r+1: n}$ and $X_{n-r: n}$ is

$$
\begin{aligned}
f\left(x_{r+1: n}, x_{n-r: n}\right) & =\frac{n !}{r !(n-2 r-2) ! r !}\left\{F\left(x_{r+1: n}\right)\right\}^{r} \\
& \times\left\{F\left(x_{n-r: n}\right)-F\left(x_{r+1: n}\right)\right\}^{n-2 r-2}\left\{1-F\left(x_{n-r: n}\right)\right\}^{r} \\
& \times f\left(x_{n-r: n}\right) f\left(x_{r+1: n}\right), \quad-\infty<X_{r+1: n}<X_{n-r: n}<\infty .
\end{aligned}
$$


Since $X_{n-r: n}=X_{r+1: n}+W$, we have

$$
\begin{aligned}
\operatorname{Pr}(W \leqslant w)= & \int_{-\infty}^{+\infty} \int_{x_{r+1: n}}^{x_{r+1: n}+w} f\left(x_{r+1: n}, x_{n-r: n}\right) d x_{n-r: n} d x_{r+1: n} \\
= & \frac{n !}{r !(n-2 r-2) ! r !} \int_{-\infty}^{+\infty}\{F(x)\}^{r} f(x) \\
& \times\left[\int_{0}^{x+w}\{1-F(u)\}^{r}\{F(u)-F(x)\}^{n-2 r-2} f(u) d u\right] d x \\
= & \frac{n !}{r !(n-2 r-2) ! r !} \int_{-\infty}^{+\infty}\{F(x)\}^{r} f(x) \\
& {\left[\int_{F(x)}^{F(x+w)}(1-y)^{r}\{y-F(x)\}^{n-2 r-2} d y\right] d x . }
\end{aligned}
$$

Integrating the expression in braces $r$ times by parts, we have

$$
\begin{aligned}
\operatorname{Pr}(W \leqslant w)= & \sum_{k=0}^{r} \prod_{i=0}^{2 r-k} \frac{(n-i)}{r !(r-k) !} \int_{-\infty}^{+\infty}\{F(x)\}^{r}\{1-F(x+w)\}^{r-k} \\
& \{F(x+w)-F(x)\}^{n-2 r+k-1} f(x) d x \\
= & \sum_{k=0}^{r} \prod_{i=0}^{2 r-k} \frac{(n-i)}{r !(r-k) !} \sum_{j=0}^{n-2 r+k-1}(-1)^{j} \\
& \left(\begin{array}{c}
n-2 r+k-1 \\
j
\end{array}\right) \sum_{l=0}^{r-k}(-1)^{l}\left(\begin{array}{c}
r-k \\
l
\end{array}\right) \\
& \times \int_{-\infty}^{\infty}\{F(x)\}^{r+j}\{F(x+w)\}^{n-2 r+k-j+l-1} f(x) d x .
\end{aligned}
$$

Let $\Lambda=\int_{-\infty}^{\infty}\{F(x)\}^{r+j}\{F(x+w)\}^{n-2 r+k-j+l-1} f(x) d x$, using $f(x ; \lambda, p)$ and $F(x ; \lambda, p)$ shown in equations (1) and (2) respectively we have

$$
\begin{aligned}
\Lambda= & \int_{-\infty}^{+\infty}\left(\frac{\lambda}{\lambda+e^{-x}}\right)^{p(r+j)}\left(\frac{\lambda}{\lambda+e^{-x-w}}\right)^{p(n-2 r+k-j+l-1)} \\
& \times \frac{p \lambda^{p} e^{-x}}{\left(\lambda+e^{-x}\right)^{p+1}} d x \\
= & \int_{-\infty}^{+\infty} \frac{p \lambda^{p(n-r+k+l)} e^{-x}}{\left(\lambda+a e^{-x}\right)^{p(n-2 r+k-j+l-1)}\left(\lambda+e^{-x}\right)^{p r+p j+p+1}} d x
\end{aligned}
$$


Let $t=\left(\lambda+a e^{-x}\right)^{-1}$, where $a=e^{-w}$, then

$$
\Lambda=p a^{p(r+j+1)} \lambda^{p(n-r+k+l)} \int_{0}^{\frac{1}{\lambda}} \frac{t^{p n-p r+p k+p l-1}}{\{1+t \lambda(a-1)\}^{p r+p j+p+1}} d t .
$$

Taking $\lambda(a-1)=b$ and $1+b t=u$, we have

$$
\begin{aligned}
& \Lambda=\frac{-p a^{p(r+j+1)}}{\left(1-e^{-w}\right)^{p(n-r+k+l)}} \\
& \times \int_{1}^{1+\frac{b}{\lambda}}(1-u)^{p n-p r+p k+p l-1} u^{-(p r+p j+p+1)} d u \text {. } \\
& =\frac{-p a^{p(r+j+1)}}{\left(1-e^{-w}\right)^{p(n-r+k+l)}} \\
& \times \int_{1}^{1+\frac{b}{\lambda}} \sum_{m=0}^{p n-p r+p k+p l-1}(-1)^{m}\left(\begin{array}{c}
p n-p r+p k+p l-1 \\
m
\end{array}\right) u^{m-p r-p j-p-1} d u \\
& =\frac{-p a^{p(r+j+1)}}{\left(1-e^{-w}\right)^{p(n-r+k+l)}} \\
& \times \int_{1}^{1+\frac{b}{\lambda}}\left\{(-1)^{p(r+j+1)}\left(\begin{array}{c}
p n-p r+p k+p l-1 \\
p r+p j-p
\end{array}\right) u^{-1}\right. \\
& \left.+\sum_{m=0, m \neq p(r+j+1)}^{p n-p r+p k+p l-1}(-1)^{m}\left(\begin{array}{c}
p n-p r+p k+p l-1 \\
m
\end{array}\right) u^{m-p r-p j-p-1}\right\} d u \\
& =\frac{p e^{-w p(r+j+1)}}{\left(1-e^{-w}\right)^{p(n-r+k+l)}}\left\{(-1)^{p(r+j+1)}\left(\begin{array}{c}
p n-p r+p k+p l-1 \\
p r+p j-p
\end{array}\right) w\right. \\
& \left.+\sum_{m=0, m \neq p(r+j+1)}^{p n-p r+p k+p l-1}(-1)^{m+1}\left(\begin{array}{c}
p n-p r+p k+p l-1 \\
m
\end{array}\right) \frac{e^{-w(m-p r-p j-p)}-1}{m-p r-p j-p}\right\} .
\end{aligned}
$$

Finally,

$$
\begin{aligned}
\operatorname{Pr}(W \leqslant w)= & \sum_{k=0}^{r} \prod_{i=0}^{2 r-k} \frac{(n-i)}{r !(r-k) !} \sum_{j=0}^{n-2 r+k-1}(-1)^{j}\left(\begin{array}{c}
n-2 r+k-1 \\
j
\end{array}\right) \\
& \times \sum_{l=0}^{r-k}(-1)^{l}\left(\begin{array}{c}
r-k \\
l
\end{array}\right) \cdot \frac{p e^{-w p(r+j+1)}}{\left(1-e^{-w}\right)^{p(n-r+k+l)}}
\end{aligned}
$$




$$
\begin{aligned}
& \times\left\{(-1)^{p(r+j+1)}\left(\begin{array}{c}
p n-p r+p k+p l-1 \\
p r+p j-p
\end{array}\right) w\right. \\
& \left.+\sum_{m=0, m \neq p(r+j+1)}^{p n-p r+p k+p l-1}(-1)^{m+1}\left(\begin{array}{c}
p n-p r+p k+p l-1 \\
m
\end{array}\right) \frac{e^{-w(m-p r-p j-p)}-1}{m-p r-p j-p}\right\} .
\end{aligned}
$$

It should be noted also that the distribution function of quasi-range of the extended type I generalized logistic distribution is free of the parameter $\lambda$. When $p=1$, the result obtained agrees with Malik (1980) for the standard logistic distribution.

\section{Acknowledgment}

The author would like to express his sincere thanks to the Head and management of the Department of Statistics and Actuarial Science, Stellenbosch University, Stellenbosch, South Africa for awarding post-doctoral fellowship to him.

\section{References}

Balakrishnan, N. and Leung, M.N. (1988a). Order statistics from the Type I generalized Logistic Distribution, Communications in Statistics - Simulation and Computation, 17, 2550 .

Balakrishnan, N. and Leung, M.Y. (1988b). Means, variances and covariances of order statistics, BLUE's for the Type I generalized logistic distribution and some applications, Communications in Statistics-Simulation and Computation, 17, 51-84.

Berkson, J. (1944). Application of the logistic function to bioassay, Journal of the American Statistical Association, 39, 357-365.

Berkson, J. (1950). Why I prefer logits to probits, Biometrics, 7, 327-339.

Berkson, J. (1953). A statistically precise and relatively simple method of estimating the bioassay and quantal response, based on the logistic function, Journal of the American Statistical Association, 48, 565-599.

Berkson, J. and Hodges, J.L. (1960). A minimax estimator for the logistic function. Fourth Berkeley Symp. Math. Stat. Prob. 4, 77-86, Berkeley- Los Angeles: University of California Press. 
Birnbaum, A. and Dudman, J. (1963). Logistic order statistics, Annals of Mathematical Statistics, 34, 658-663.

David, H.A. (1970). Order Statistics. Wiley, New York.

George, E.O. and Ojo, M.O. (1980). On a generalization of the logistic distribution, Annals of Statistical Mathematics, 32, 161-169.

Gupta, S.S., Qureishi, A.S. and Shah, B.K. (1967). Best linear unbiased estimators of the parameters of the logistic distribution using order statistics, Technometrics, 9, 43-56.

Gupta, S.S. and Shah, B.K. (1965). Exact moments and percentage points of the order statistics and the distribution of the range from the logistic distribution. Annals of Mathematical Statistics, 36, 907-920.

Malik, H.J. (1980). Exact formula for the cumulative distribution function of the quasi-range from the logistic distribution. Commun. Statist.-Theor. Meth., 14, 1527-1534.

Ojo, M.O. (1989). Analysis of some prison data. Journal of applied statistics, 16, 377-383.

Olapade, A.K. (2004). On extended type I generalized logistic distribution. International Journal of Mathematics and Mathematical Sciences. 57, 3069-3074.

Olapade, A.K. (2005). On negatively skewed extended generalized logistic distribution, Kragujevac J. Math., 27, 175-182.

Olapade, A.K. (2006). On symmetric extended generalized logistic distribution. Journal of Statistical Research of Iran, 3, 113-137.

Olapade, A.K. (2009). Some properties and application of the extended type I generalized logistic distribution. ICASTOR Journal of Mathematical Sciences, 3, 73-83.

Plackett, R.L. (1958). Linear estimation from censored data, Ann. Math. Statist. 29, 131-142.

Shah, B.K. (1966). On the bivariate moments of order statistics from a logistic distribution, Annals of Mathematical Statistics, 37, 1002-1010.

Shah, B.K. (1970). Note on moments of a logistic order statistics, Annals of Mathematical Statistics, 41, 2151-2152.

Tarter, M.E. and Clark, V.A. (1965). Properties of the median and other order statistics of logistic variates. Annals of Mathematical Statistics, 36, 1779-1786.

Wu Jong-Wuu, Hung Wen-Liang and Lee Hsiu-Mei (2000). Some Moments and Limit Behaviors of the Generalized Logistic Distribution with Applications. Proc. Natl. Sci. Counc. $\operatorname{ROC}(4), \mathbf{2 4}, 7-14$. 


\section{A. K. Olapade}

Mathematics Department,

Obafemi Awolowo University,

Ile-Ife,

220005 ,

Nigeria.

email: akolapad@oauife.edu.ng

olapade@sun.ac.za 